lustitia Socialis. Revista Arbitrada de Ciencias Jurídicas.

Año V. Vol. V. №3. Edición Especial. 2020-III:

Universidad Regional Autónoma de los Andes

Hecho el depósito de Ley: FA2016000064 ISSN: 2542-3371

FUNDACIÓN KOINONIA (F.K). Santa Ana de Coro, Venezuela

Milena Elizabeth Álvarez-Tapia; Pablo David Portilla-Obando

http://dx.doi.org/10.35381/racii.v5i3.1141

\title{
El impacto social y jurídico sobre la movilidad inclusiva de personas con discapacidad
}

The social and legal impact on inclusive mobility of people with disabilities

\author{
Milena Elizabeth Álvarez-Tapia \\ ut.milenaalvarez@uniandes.edu.ec \\ Universidad Regional Autónoma de los Andes, Tulcán \\ Ecuador \\ https://orcid.org/0000-0003-3143-9342 \\ Pablo David Portilla-Obando \\ Universidad Regional Autónoma de los Andes, Tulcán \\ Ecuador \\ pabloportilla70@gmail.com
}

Recibido: 31 de octubre de 2020

Revisado: 10 de octubre de 2020

Aprobado: 05 de diciembre de 2020

Publicado: 10 de diciembre de 2020 
lustitia Socialis. Revista Arbitrada de Ciencias Jurídicas.

Año V. Vol. V. N³. Edición Especial. 2020-III:

Universidad Regional Autónoma de los Andes

Hecho el depósito de Ley: FA2016000064 ISSN: 2542-3371

FUNDACIÓN KOINONIA (F.K). Santa Ana de Coro, Venezuela

Milena Elizabeth Álvarez-Tapia; Pablo David Portilla-Obando

\title{
RESUMEN
}

La investigación tuvo por objetivo analizar el impacto social y jurídico sobre la movilidad inclusiva de personas con discapacidad. Se desarrolló desde el enfoque cuantitativo y metodológicamente desde un tipo descriptiva con diseño de campo no experimental transversal, recopilándose información en único momento en una población de 40 personas con discapacidad de Tulcán - Ecuador. Aunque la legislación constitucional como norma jerárquicamente superior prioriza a las personas con discapacidad, en la adquisición de sus derechos y el mejoramiento en todos los ámbitos sociales, educativos, de salud, de trabajo e inclusión de movilidad, se ha podido esclarecer la problemática que radica en la ciudad de Tulcán, debido a que aún no se han instaurado políticas públicas para la sensibilización de la movilidad y accesibilidad de las personas con discapacidad, sea en instituciones públicas y privadas, la mayoría aun no goza de los medios adecuados que están garantizados para su movilidad de independencia.

Descriptores: Discriminación; violación de los derechos humanos; derecho al trabajo. (Palabras tomadas del Tesauro UNESCO).

\begin{abstract}
The objective of the research was to analyze the social and legal impact on the inclusive mobility of people with disabilities. It was developed from a quantitative approach and methodologically from a descriptive type with a non-experimental cross-sectional field design, collecting information at a single moment in a population of 40 people with disabilities from Tulcán - Ecuador. Although constitutional legislation as a hierarchically superior norm prioritizes people with disabilities, in the acquisition of their rights and improvement in all social, educational, health, work and mobility inclusion areas, it has been possible to clarify the problem that lies In the city of Tulcán, due to the fact that public policies have not yet been established to raise awareness of the mobility and accessibility of people with disabilities, both in public and private institutions, the majority still do not have the adequate means that are guaranteed to their mobility of independence.
\end{abstract}

Descriptors: Discrimination; Human rights violations; Right to employment. (Words taken from the UNESCO Thesaurus). 
lustitia Socialis. Revista Arbitrada de Ciencias Jurídicas.

Año V. Vol. V. N³. Edición Especial. 2020-III:

Universidad Regional Autónoma de los Andes

Hecho el depósito de Ley: FA2016000064

ISSN: 2542-3371

FUNDACIÓN KOINONIA (F.K). Santa Ana de Coro, Venezuela

Milena Elizabeth Álvarez-Tapia; Pablo David Portilla-Obando

\section{INTRODUCCIÓN}

La exigencia de los derechos de las personas con discapacidad se ha visto en vulnerabilidad ante el rechazo y la discriminación entorno a su vida social y afectiva sobre sus condiciones, a pesar de que en la actualidad se lo ha denominado por la Constitución Ecuatoriana del 2008 como un grupo de atención prioritaria y vulnerable ante ciertas circunstancias que, para la sociedad en lo común, son de fácil accesibilidad debido a una capacidad física estable.

La Ley Orgánica de Discapacidades define a la persona titular de derechos conforme a la inserción social de movilidad, en el Artículo 86 de la Ley Orgánica de Discapacidades, que determina que las personas con discapacidad tienen derecho a la protección y promoción social por parte del Estado, que les permita el máximo desarrollo de su personalidad, fomento de autonomía y la disminución de la dependencia.

Es así como, es necesario que el fomento de su autonomía y disminución de dependencia, este dirigido y reflejado en lo más importante, como es el acceso a cualquier institución laboral o educativa, que, bajo las condiciones de movilidad en la ciudad de Tulcán, algunas de estas no cuentan con este mecanismo de fomento y protección.

Un sistema sustentable y equitativo, debe asegurar que todas las personas tengan acceso a distintos modos de transporte de manera segura, indiferentemente de su clase social, su identidad de género, su clase social o su condición física.

Los Estados han contemplado la movilidad de las personas con discapacidad a través de las inversiones, los presupuestos y la planificación de los sistemas de transporte público y el acceso a instituciones públicas o privadas que carecen de mecanismos de accesibilidad, y si en algunas se han instaurado han sido realizadas de forma ineficiente al contemplar ya la población que está relacionada específicamente en la ciudad de Tulcán, El resultado ha sido experiencias deprimentes en detrimento de otras áreas de trabajo importantes a cargo de la municipalidad como son el proveer condiciones adecuadas de habitabilidad, convivencia, el medio ambiente, la alimentación, la salud y 
lustitia Socialis. Revista Arbitrada de Ciencias Jurídicas.

Año V. Vol. V. N³. Edición Especial. 2020-III:

Universidad Regional Autónoma de los Andes

Hecho el depósito de Ley: FA2016000064

ISSN: 2542-3371

FUNDACIÓN KOINONIA (F.K). Santa Ana de Coro, Venezuela

Milena Elizabeth Álvarez-Tapia; Pablo David Portilla-Obando

la educación, entre otros.

Eso no solo es la afectación en la ciudad Tulcán, a lo largo de muchas ciudades en territorio ecuatoriano y nivel de Latinoamérica, el hacinamiento, la desprotección, el mal servicio y el acoso son solo algunos de los deplorables denominadores comunes en la accesibilidad de movilidad de las personas con discapacidad.

\section{MÉTODO}

La investigación se desarrolló primordialmente desde el enfoque cuantitativo y metodológicamente desde un tipo descriptiva con diseño de campo no experimental transversal, recopilándose información en único momento en una población de 40 personas con discapacidad de la población de Tulcán - Ecuador, a quienes se les aplicó una encuesta y cuestionario de alternativas dicotómicas, siendo validado por juicio de expertos y cálculo de coeficiente Kuder-Richardson (KR-20) con un valor de 9,79 siendo confiable para su aplicación. Los datos recopilados fueron procesados estadísticamente desde la perspectiva descriptiva, presentándose en frecuencias y porcentajes los resultados.

\section{RESULTADOS}

Se presentan los resultados a partir del análisis estadístico desarrollado:

\section{Tabla 1.}

¿Cómo profesional del derecho conoce usted los derechos primordiales de las personas con discapacidad?.

\begin{tabular}{ccc}
\hline Alternativas & Personas & Porcentaje \\
\hline SI & 40 & $100 \%$ \\
NO & 0 & $0 \%$ \\
\hline TOTAL & 40 & $100 \%$ \\
\hline
\end{tabular}


lustitia Socialis. Revista Arbitrada de Ciencias Jurídicas.

Año V. Vol. V. N³. Edición Especial. 2020-III:

Universidad Regional Autónoma de los Andes

Hecho el depósito de Ley: FA2016000064

ISSN: 2542-3371

FUNDACIÓN KOINONIA (F.K). Santa Ana de Coro, Venezuela

Milena Elizabeth Álvarez-Tapia; Pablo David Portilla-Obando

Ante la formulación de esta pregunta dirigida a funcionarios y profesionales del derecho en libre ejercicio y bajo dependencia del cantón Tulcán; se puede comprobar que de las 40 personas encuestadas si conocen de los derechos primordiales de las personas con discapacidad.

Tabla 2.

¿Usted como profesional del derecho ha observado alguna vulneración con respecto a las personas con discapacidad?

\begin{tabular}{ccc}
\hline Alternativas & Personas & Porcentaje \\
\hline SI & 40 & $100 \%$ \\
NO & 0 & $0 \%$ \\
\hline TOTAL & 40 & $100 \%$ \\
\hline
\end{tabular}

Ante la formulación de esta pregunta dirigida a funcionarios y profesionales del derecho en libre ejercicio y bajo dependencia del cantón Tulcán; se puede comprobar que de las 40 personas encuestadas han observado y palpado en alguna circunstancia la realidad de alguna vulneración en afectación a las personas con discapacidad.

\section{Tabla 3.}

¿Está de acuerdo que se implementen los mecanismos correctos de accesibilidad en razón a la movilidad independiente de las personas con discapacidad?.

\begin{tabular}{ccc}
\hline Alternativas & Personas & Porcentaje \\
\hline SI & 40 & $100 \%$ \\
NO & 0 & $0 \%$ \\
\hline TOTAL & 40 & $100 \%$ \\
\hline
\end{tabular}


lustitia Socialis. Revista Arbitrada de Ciencias Jurídicas.

Año V. Vol. V. N³. Edición Especial. 2020-III:

Universidad Regional Autónoma de los Andes

Hecho el depósito de Ley: FA2016000064

ISSN: 2542-3371

FUNDACIÓN KOINONIA (F.K). Santa Ana de Coro, Venezuela

Milena Elizabeth Álvarez-Tapia; Pablo David Portilla-Obando

Ante la formulación de esta pregunta dirigida a funcionarios y profesionales del derecho en libre ejercicio y bajo dependencia del cantón Tulcán; se puede comprobar que de las 40 personas encuestadas están de acuerdo en que se implementen mecanismos correctos de accesibilidad en razón a la movilidad independiente de las personas con discapacidad.

\section{Tabla 4.}

¿Considera necesario la implementación e inspección regular por parte del Consejo Nacional para la Igualdad de Discapacidades, con respecto al cumplimiento de instituciones pública y privadas en la accesibilidad de sus servicios para personas con discapacidad?

\begin{tabular}{ccc}
\hline Alternativas & Personas & Porcentaje \\
\hline SI & 40 & $100 \%$ \\
NO & 0 & $0 \%$ \\
\hline TOTAL & 40 & $100 \%$ \\
\hline
\end{tabular}

Ante la formulación de esta pregunta dirigida a funcionarios y profesionales del derecho en libre ejercicio y bajo dependencia del cantón Tulcán; se puede comprobar que de las 40 personas encuestadas si consideran necesario la implementación e inspección de regulación por parte del Consejo Nacional para la Igualdad de Discapacidades, con respecto al cumplimiento de instituciones públicas y privadas ante la falta de accesibilidad de sus servicios para las personas con discapacidad. 
lustitia Socialis. Revista Arbitrada de Ciencias Jurídicas.

Año V. Vol. V. N³. Edición Especial. 2020-III:

Universidad Regional Autónoma de los Andes

Hecho el depósito de Ley: FA2016000064 ISSN: 2542-3371

FUNDACIÓN KOINONIA (F.K). Santa Ana de Coro, Venezuela

Milena Elizabeth Álvarez-Tapia; Pablo David Portilla-Obando

\section{DISCUSIÓN}

La inclusión social de las personas con discapacidad, tiene su esencia en respetar sus derechos fundamentales, en el caso de la educación universitaria, no basta con asignar un cupo, es necesario reformar el currículo y los centros educativos para que pueda desarrollarse una interacción docente - estudiantes, en razón de salvaguardar el mayor beneficio de la persona con discapacidad (Pérez-Castro, 2016), sin embargo, (PérezCastro, 2019), advierte que a pesar que las políticas universitarias han disminuido la brecha, aún no han logrado el ser efectiva en una masificación de las personas con discapacidad al ámbito de las universidades, situación que debe ser revisada con la finalidad de proyectar estrategias de mayor efectividad.

El derecho al trabajo es un tema transversal cuando se habla de inclusión social en personas con discapacidad, los empleadores se enfrentan al dilema de contratar o no, según el grado de discapacidad y de tipo de trabajo a realizar, (Alcover \& Pérez-Torres, 2011), destacan que aquellos ligados al teletrabajo y atención al público, son los de mayor factibilidad de asumir, siendo una adecuada formación escolar - profesional en este sentido, para que no solo logren un aprendizaje formal, sino, compaginado a lo laboral.

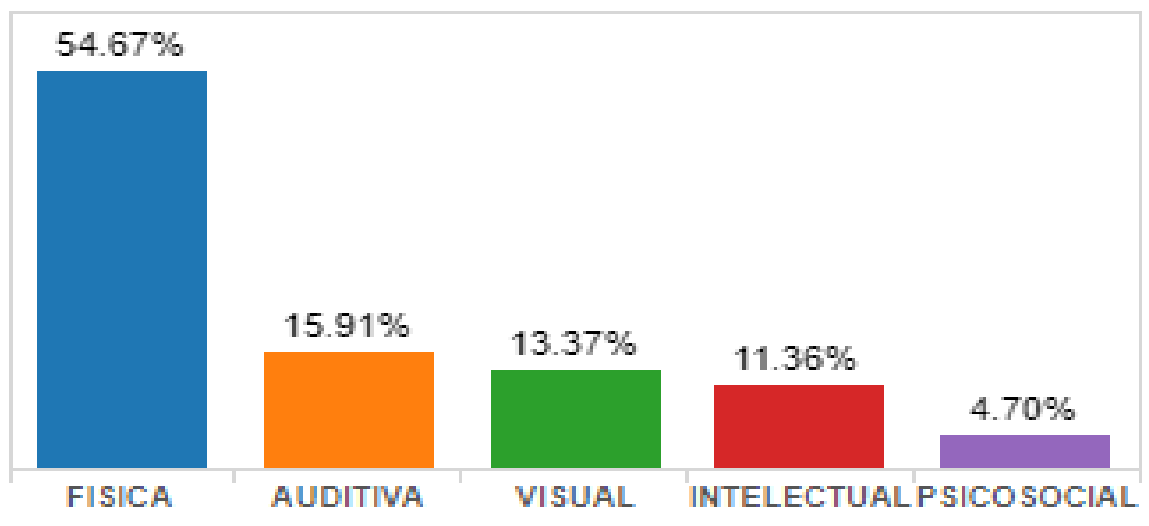

Figura 1. Tipos de discapacidad en personas en el sector laboral.

Fuente: Consejo nacional para la igualdad de discapacidades (2020). 
lustitia Socialis. Revista Arbitrada de Ciencias Jurídicas.

Año V. Vol. V. N³. Edición Especial. 2020-III:

Universidad Regional Autónoma de los Andes

Hecho el depósito de Ley: FA2016000064

ISSN: 2542-3371

FUNDACIÓN KOINONIA (F.K). Santa Ana de Coro, Venezuela

Milena Elizabeth Álvarez-Tapia; Pablo David Portilla-Obando

La mayor proporcionalidad se encuentra ligada a discapacidades físicas, lo cual a juicio de (Gómez-Tagle-López, et al., 2016), puede verse influenciado por el hecho de que aún se concibe como paradigma social, que el discapacitado porta una enfermedad mental, lo cual se relaciona con el hecho de recibir asistencia especializada, mientras que se debe pensar en un modelo integrador, donde se valore al discapacitado como una persona que puede aprender constantemente a desempeñarse en la sociedad y en el campo laboral - educativo.

En este sentido, en el Ecuador, en los últimos años se han tomado correctivos en la legislación con la finalidad de corregir la escasa o nula participación socioeconómica de las personas con discapacidad (Arteaga, 2019), lo cual no debería ser un caso de exclusión laboral, por cuanto esto corrompe el derecho universal (Nuvaez-Castillo, 2019), con lo cual se desarrollarían brechas sociales y judiciales en detrimento de la persona, siendo esto un tipo de violencia que debe ser erradicado, sobre todo cuando la víctima es mujer (Aldana-Zavala \& Valles-González, 2018).

Así mismo, (Gallegos-Erazo \& Mantilla-Buenaño, 2018), consideran que a pesar de los avances legislativos desarrollados en favor de las personas con discapacidad, se requiere profundizar la formación social en relación a percibir con una visión equitativa e incluyente a las personas con discapacidad, de ese modo, podrá reducirse la brecha discriminatoria en detrimento de este segmento poblacional, aunque desde el trabajo de (Friend-Macías \& Álava-Díaz, 2019), se debe profundizar en el plano contractual entre las personas con discapacidad y los ofertantes de bienes y servicios, esto con la finalidad de corregir falencias jurídicas en tal sentido.

\section{CONCLUSIÓN}

La realidad social y jurídica que ha llevado a la vulneración de movilidad e inserción social a personas con discapacidad de la ciudad de Tulcán, y que a través de la fundamentación teórica, doctrinaria y jurídica se ha podido establecer mediante la legislación nacional e internacional que la afectación de independencia en personas con discapacidad al 
lustitia Socialis. Revista Arbitrada de Ciencias Jurídicas.

Año V. Vol. V. N³. Edición Especial. 2020-III:

Universidad Regional Autónoma de los Andes

Hecho el depósito de Ley: FA2016000064 ISSN: 2542-3371

FUNDACIÓN KOINONIA (F.K). Santa Ana de Coro, Venezuela

Milena Elizabeth Álvarez-Tapia; Pablo David Portilla-Obando

respecto de su accesibilidad en el recorrido y movilidad en la adquisición del uso de servicios de instituciones públicas y privadas, se ha visto en vulneración a través de fundamentos históricos de la evolución del ser humano, el fomento de la discriminación y la desigualdad social de las personas con discapacidad.

Aunque la legislación constitucional como norma jerárquicamente superior prioriza a las personas con discapacidad, en la adquisición de sus derechos y el mejoramiento en todos los ámbitos sociales, educativos, de salud, de trabajo e inclusión de movilidad, se ha podido esclarecer la problemática que radica en la ciudad de Tulcán, debido a que aún no se han instaurado políticas públicas para la sensibilización de la movilidad y accesibilidad de las personas con discapacidad, sea en instituciones públicas y privadas, la mayoría aun no goza de los medios adecuados que están garantizados para su movilidad de independencia.

\section{FINANCIAMIENTO}

No monetario.

\section{AGRADECIMIENTO}

A la Universidad Regional Autónoma de Los Andes; por motivar el desarrollo de la Investigación.

\section{REFERENCIAS CONSULTADAS}

Pérez-Castro, J. (2016). La inclusión de las personas con discapacidad en la educación superior en México [The inclusion of people with disabilities in higher education in Mexico]. Sinéctica, Revista Electrónica de Educación, (46),1-15.

Pérez-Castro, J. (2019). La inclusión de los estudiantes con discapacidad en dos universidades públicas mexicanas [Inclusion of students with disabilities in two Mexican public universities]. Innovación educativa (México, DF), 19(79), 145-170. 
lustitia Socialis. Revista Arbitrada de Ciencias Jurídicas.

Año V. Vol. V. N³. Edición Especial. 2020-III:

Universidad Regional Autónoma de los Andes

Hecho el depósito de Ley: FA2016000064 ISSN: 2542-3371

FUNDACIÓN KOINONIA (F.K). Santa Ana de Coro, Venezuela

Milena Elizabeth Álvarez-Tapia; Pablo David Portilla-Obando

Alcover, C, \& Pérez-Torres, V. (2011). Trabajadores con discapacidad: problemas, retos y principios de actuación en salud ocupacional [Workers with disabilities: Concerns, challenges, and intervention principles in occupational health]. Medicina y Seguridad del Trabajo, 57(Supl. 1), 206-223. https://dx.doi.org/10.4321/S0465$\underline{546 \times 2011000500013}$

Consejo nacional para la igualdad de discapacidades. (2020). Tipos de discapacidad. Recuperado de https://n9.cl/rOf9

Gómez-Tagle-López, E, \& Castillo-Fernández, D. (2016). Sociología de la discapacidad [Sociology of Disability]. Tla-melaua, 10(40), 176-194.

Arteaga, A. (2019). Inclusión laboral de las personas con discapacidad [Labor inclusion of people with disabilities]. IUSTITIA SOCIALIS, 4(1), 16-38. http://dx.doi.org/10.35381/racii.v4i1.536

Nuvaez-Castillo, J. (2019). Alcance sociocultural de la discriminación laboral en razón del género y la edad [Sociocultural scope of employment discrimination based on gender and age]. IUSTITIA SOCIALIS, 4(6), 4-27. http://dx.doi.org/10.35381/racji.v4i6.286

Aldana-Zavala, J., \& Valles-González, E. (2018). La mujer en Derecho a una Vida libre de violencia desde la Organización Comunitaria [Women in the Right to a Life Free of Violence from the Community Organization]. IUSTITIA SOCIALIS, 3(5), 110127. http://dx.doi.org/10.35381/racji.v3i5.171

Gallegos-Erazo, F., \& Mantilla-Buenaño, G. (2018). Hacia la igualdad e inclusión laboral de las personas con discapacidad en Ecuador [Towards equality and labor inclusion of people with disabilities in Ecuador]. Yachana Revista Científica, 6(2), 48-60.

Friend-Macías, R. A., \& Álava-Díaz, M. (2019). La capacidad jurídica de los discapacitados intelectuales y sus derechos como consumidores en Ecuador según la Convención de los derechos de las personas con discapacidad [The legal capacity of the intellectually disabled and their rights as consumers in Ecuador according to the Convention on the Rights of Persons with Disabilities]. USFQ Law Review, 6(1), 131-146. https://doi.org/10.18272//r.v6i1.1384 
lustitia Socialis. Revista Arbitrada de Ciencias Jurídicas.

Año V. Vol. V. №3. Edición Especial. 2020-III:

Universidad Regional Autónoma de los Andes

Hecho el depósito de Ley: FA2016000064

ISSN: 2542-3371

FUNDACIÓN KOINONIA (F.K). Santa Ana de Coro, Venezuela

\section{Milena Elizabeth Álvarez-Tapia; Pablo David Portilla-Obando}

(C)2020 por los autores. Este artículo es de acceso abierto y distribuido según los términos y condiciones de la licencia Creative Commons Atribución-NoComercial-Compartirlgual 4.0 Internacional (CC BY-NC-SA 4.0)

(https://creativecommons.org/licenses/by-nc-sa/4.0/). 